\title{
Design and Implementation of Cryptographic Algorithm Based on Reactive Elements and RNA Codons for Secured Transmission
}

\author{
Saswata Dasgupta \\ Department of Computer Sc. \& Engineering \\ JIS College of Engineering, Kalyani,West Bengal, India \\ Kumar Gaurav Verma \\ Department of Computer Sc. \& Engineering \\ JIS College of Engineering, Kalyani,West Bengal, India \\ Sudipta Sahana \\ Assistant Professor, Department of Computer Sc. \& Engineering \\ JIS College of Engineering, Kalyani,West Bengal, India \\ Rajdeep Chowdhury \\ Assistant Professor, Department of Computer Application \\ JIS College of Engineering, Kalyani,West Bengal, India
}

\begin{abstract}
Network Security has become imperative in the contemporary scenario and subsequently an assortment of modus operandi is espoused to evade it. Network administrators need to adhere with latest advancement in both hardware and software field to prevent user data from malicious intrusions. The formulated paper outlines a cryptographic algorithm based on elements of Reactive Series and RNA Codons employing the identical concept amid its functionality. An amalgamation would endow with a proficient and prearranged approach of amassing data with stringent security modus operandi, with effective deployment of all obtainable space. The incorporation of the novel cryptographic algorithm would ensure performance enhancement in course of action. The pertinent employment of the formulated work is ensured in a variety of organizations where accrual of cosseted data is of extreme enormity.
\end{abstract}

Keywords - Encryption; Decryption; ASCII Table; Reactivity Series; Codon Table; RNA

\section{INTRODUCTION}

The concept of ideal confidentiality has been prevalent since the 1950s and the modus operandi of encrypting data is quite highly accepted, when the notion of security implementation comes at its premier standards. Nevertheless, over the years, the modus operandi to engender the Seed employed in such encryption techniques has only diversified with the endeavour of making a move towards a much more robust technique, to say the least. The proposed cryptographic algorithm is classified as a Stream Cipher algorithm, as the Seed is applied to apiece character at a time. The concept of Reactive Elements according to the Reactivity Series is applied in the proposed algorithm. Furthermore, the paper guides with the mechanism in which the appliance have been ensured in a part by part basis, for utmost ease in understanding. It is imperative to employ a dynamic Seed to ascertain ideal confidentiality. It would be effectual to say that the entire paper covers the prime aspect established in conceptual juncture and meets economic feasibility, viability as well as scalability. 


\section{PROPOSED ALGORITHM}

For utmost ease of understanding, the entire flowchart of the proposed work is stated below:

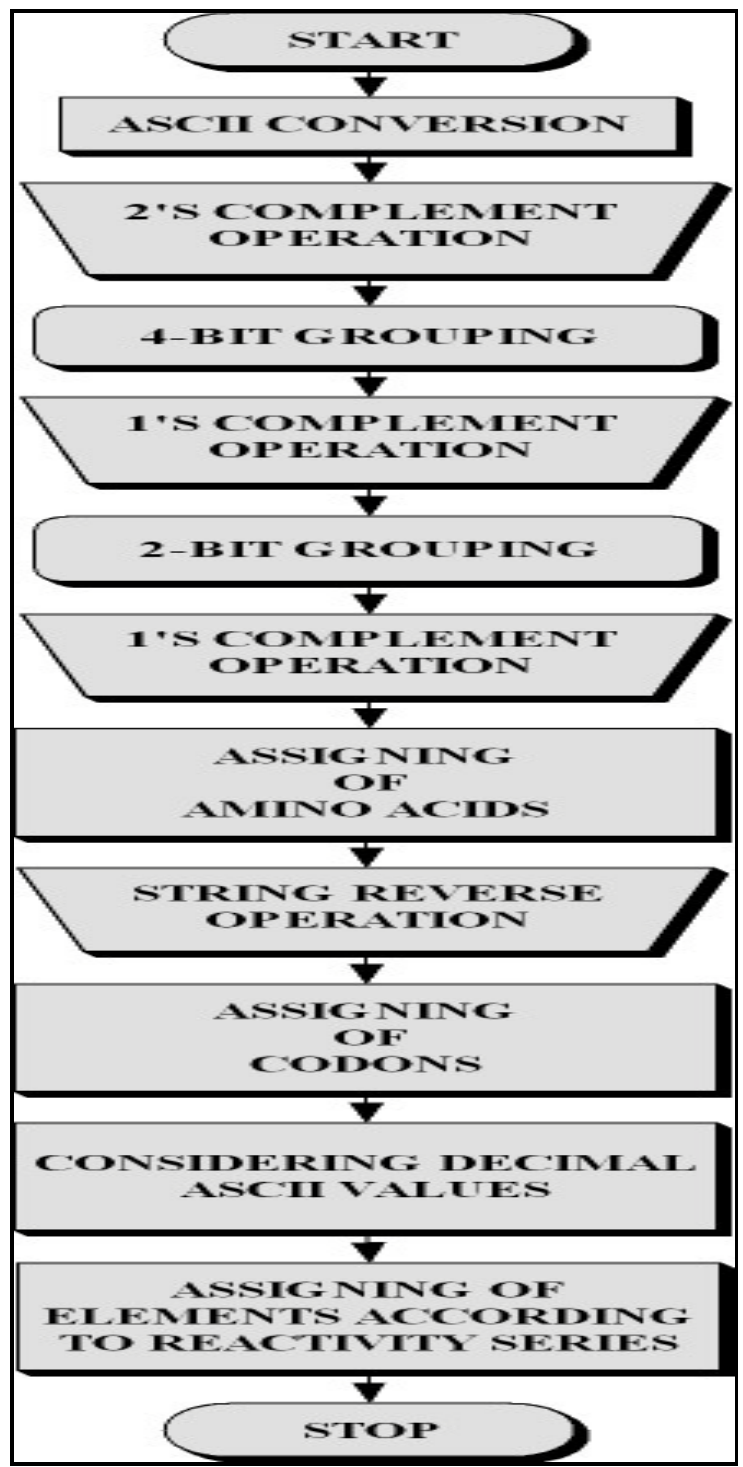

Figure-1: Flowchart of Entire Work

\section{A. Encryption -}

At the very first inception, considering the plain text to be “Test@1234” 


\begin{tabular}{|c|c|}
\hline Character & ASCII Value \\
\hline $\mathrm{T}$ & 01010100 \\
\hline $\mathrm{e}$ & 01100101 \\
\hline $\mathrm{s}$ & 01110011 \\
\hline $\mathrm{t}$ & 01110100 \\
\hline $\mathrm{Q}$ & 01000000 \\
\hline 1 & 00000001 \\
\hline 2 & 00000010 \\
\hline 3 & 00000011 \\
\hline 4 & 00000100 \\
\hline
\end{tabular}

Step 1: Apiece character of the plain text is converted to its corresponding 8-bit ASCII Value.

\begin{tabular}{|c|cc|}
\hline 8-bit ASCII & \multicolumn{2}{|c|}{ 4-bit Grouping } \\
\hline 10101100 & 1010 & 1100 \\
\hline 10011011 & 1001 & 1011 \\
\hline 10001101 & 1000 & 1100 \\
\hline 10001100 & 1000 & 1100 \\
\hline 11000000 & 1100 & 0000 \\
\hline 11111111 & 1111 & 1111 \\
\hline 11111110 & 1111 & 1110 \\
\hline 11111101 & 1111 & 1101 \\
\hline 11010110 & 1101 & 0110 \\
\hline
\end{tabular}

Step 3: Grouping of 4 bits of segments is ensured.

\begin{tabular}{|ll|llll|}
\hline \multicolumn{3}{|c|}{ 4-bit Groups } & \multicolumn{3}{|c|}{ 2-bit Grouping } \\
\hline 0101 & 0011 & 01 & 01 & 00 & 11 \\
\hline 0110 & 0100 & 01 & 10 & 01 & 00 \\
\hline 0111 & 0010 & 01 & 11 & 00 & 10 \\
\hline 0111 & 0011 & 01 & 11 & 00 & 11 \\
\hline 0011 & 1111 & 00 & 11 & 11 & 11 \\
\hline 0000 & 0000 & 00 & 00 & 00 & 00 \\
\hline 0000 & 0001 & 00 & 00 & 00 & 01 \\
\hline 0000 & 0010 & 00 & 00 & 00 & 10 \\
\hline 0010 & 1001 & 00 & 10 & 10 & 01 \\
\hline
\end{tabular}

Step 5: Segments of 2 bit Grouping is ensured.

\begin{tabular}{|c|c|}
\hline ASCII Value & 2's Complement \\
\hline 01010100 & 10101100 \\
\hline 01100101 & 10011011 \\
\hline 01110011 & 10001101 \\
\hline 01110100 & 10001100 \\
\hline 01000000 & 11000000 \\
\hline 00000001 & 11111111 \\
\hline 00000010 & 11111110 \\
\hline 00000011 & 11111101 \\
\hline 00000100 & 11010110 \\
\hline
\end{tabular}

Step 2: Apiece 8-bit ASCII Value is operated with 2's Complement.

\begin{tabular}{|cc|cc|}
\hline \multicolumn{2}{|c|}{ 4-bit Groups } & \multicolumn{2}{|c|}{ 1's Complement } \\
\hline 1010 & 1100 & 0101 & 0011 \\
\hline 1001 & 1011 & 0110 & 0100 \\
\hline 1000 & 1100 & 0111 & 0010 \\
\hline 1000 & 1100 & 0111 & 0011 \\
\hline 1100 & 0000 & 0011 & 1111 \\
\hline 1111 & 1111 & 0000 & 0000 \\
\hline 1111 & 1110 & 0000 & 0001 \\
\hline 1111 & 1101 & 0000 & 0010 \\
\hline 1101 & 0110 & 0010 & 1001 \\
\hline
\end{tabular}

Step 4: 1's Complement operation is performed.

\begin{tabular}{|c|c|}
\hline 2-bit Groups & 1's Complement \\
\hline $\begin{array}{llll}01 & 01 & 00 & 11\end{array}$ & $\begin{array}{llll}10 & 10 & 11 & 00\end{array}$ \\
\hline $\begin{array}{llll}01 & 10 & 01 & 00\end{array}$ & $\begin{array}{llll}10 & 01 & 10 & 11 \\
\end{array}$ \\
\hline $\begin{array}{llll}01 & 11 & 00 & 10\end{array}$ & $\begin{array}{llll}10 & 00 & 11 & 01\end{array}$ \\
\hline $\begin{array}{llll}01 & 11 & 00 & 11 \\
\end{array}$ & $\begin{array}{llll}10 & 00 & 11 & 00 \\
\end{array}$ \\
\hline $\begin{array}{llll}00 & 11 & 11 & 11\end{array}$ & $\begin{array}{llll}11 & 00 & 00 & 00\end{array}$ \\
\hline $\begin{array}{llll}00 & 00 & 00 & 00\end{array}$ & $\begin{array}{llll}11 & 11 & 11 & 11 \\
\end{array}$ \\
\hline $\begin{array}{lllll}00 & 00 & 00 & 0 & 1 \\
\end{array}$ & $\begin{array}{llll}11 & 11 & 11 & 10 \\
\end{array}$ \\
\hline $\begin{array}{lllll}00 & 00 & 00 & 10 \\
\end{array}$ & $\begin{array}{llll}11 & 11 & 11 & 01 \\
\end{array}$ \\
\hline $\begin{array}{llll}00 & 10 & 10 & 01\end{array}$ & $\begin{array}{llll}11 & 01 & 01 & 10\end{array}$ \\
\hline
\end{tabular}

Step 6: Operation of 1's Complement is ensured 


\begin{tabular}{|c|c|}
\hline 2-bit Groups & Amino Acids \\
\hline $\begin{array}{lllll}10 & 10 & 11 & 00\end{array}$ & C C A U \\
\hline $\begin{array}{llll}10 & 01 & 10 & 11\end{array}$ & C G C A \\
\hline $\begin{array}{llll}10 & 00 & 11 & 01\end{array}$ & $\mathrm{C} \cup \mathrm{A} G$ \\
\hline $\begin{array}{llll}10 & 00 & 11 & 00\end{array}$ & $\mathrm{C} U \mathrm{~A} U$ \\
\hline $\begin{array}{llll}11 & 00 & 00 & 00\end{array}$ & A U U U \\
\hline $\begin{array}{llll}11 & 11 & 11 & 11\end{array}$ & A A A A \\
\hline $\begin{array}{llll}11 & 11 & 11 & 10\end{array}$ & A A A C \\
\hline $\begin{array}{llll}11 & 11 & 11 & 01\end{array}$ & A A A G \\
\hline $\begin{array}{llll}11 & 01 & 01 & 10\end{array}$ & A G G C \\
\hline
\end{tabular}

Step 7: Apiece 2-bit segments are assigned to Amino Acids U G C A, as stated below:
$00-\mathrm{U}$
$01-\mathrm{G}$
$10-\mathrm{C}$
$11-\mathrm{A}$

\begin{tabular}{|c|c|}
\hline Original Value & Reversed Value \\
\hline C C A U & U A C C \\
\hline C G C A & A C G C \\
\hline C U A G & G A U C \\
\hline C U A U & U A U \\
\hline A U U U & U U U A \\
\hline A A A A & A A A A \\
\hline A A A C & C A A A \\
\hline A A A G & G A A \\
\hline A G G & C G G A \\
\hline
\end{tabular}

Step 8: Reverse String operation is performed.

Hence, ignoring the last and the first bit simultaneously and considering the rest 3 bits for referring to the Codon Table until all segments are employed, is ensured.

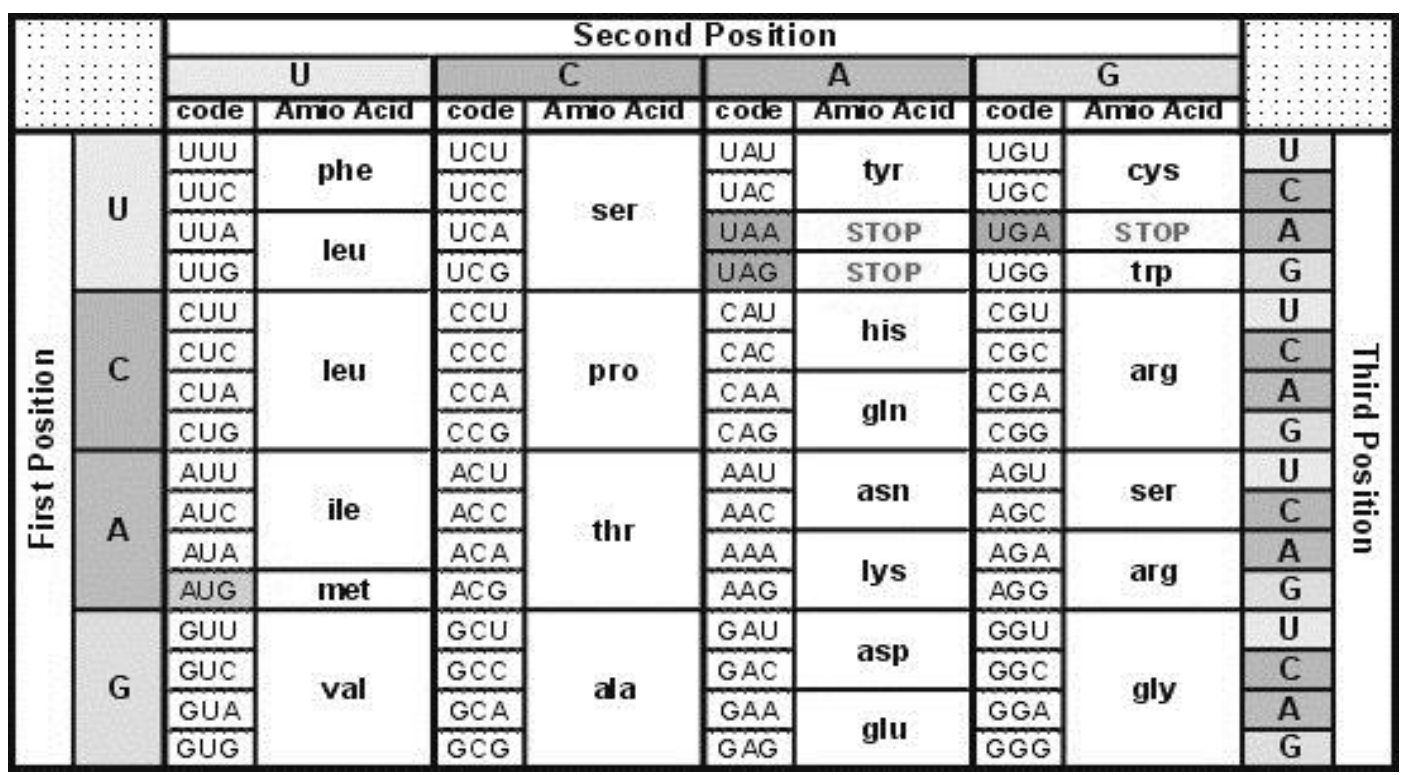

Figure-2: Codon Table

\begin{tabular}{|c|c|}
\hline Amino Acids & Codons \\
\hline U A C C & t y r C \\
\hline A C G C & A a r g \\
\hline G A U C & a s p C \\
\hline U A U C & U i e \\
\hline U U U A & p h e A \\
\hline A A A A & A l y s \\
\hline C A A A & g l n A \\
\hline G A A A & G l y s \\
\hline C G G A & a r g A \\
\hline
\end{tabular}

\begin{tabular}{|c|c|}
\hline Codons & Decimal ASCII \\
\hline t y r C & 11612111467 \\
\hline A a r g & 6597114103 \\
\hline a s p C & 9711511299 \\
\hline U i l e & 85105108101 \\
\hline p h e A & 11210410165 \\
\hline A l y s & 65108121115 \\
\hline g l n A & 10310811065 \\
\hline G l y s & 71108121115 \\
\hline a r g A & 9711410365 \\
\hline
\end{tabular}




\begin{tabular}{|c|c|}
\hline Numeric Value & $\begin{array}{c}\text { Corresponding } \\
\text { Element }\end{array}$ \\
\hline 0 & $\mathrm{~K}$ \\
\hline 1 & $\mathrm{Na}$ \\
\hline 2 & $\mathrm{Ca}$ \\
\hline 3 & $\mathrm{Mg}$ \\
\hline 4 & $\mathrm{Al}$ \\
\hline 5 & $\mathrm{Zn}$ \\
\hline 6 & $\mathrm{Fe}$ \\
\hline 7 & $\mathrm{Sn}$ \\
\hline 8 & $\mathrm{~Pb}$ \\
\hline 9 & $\mathrm{H}$ \\
\hline
\end{tabular}

\begin{tabular}{|c|c|}
\hline Decimal ASCII & Element Segments \\
\hline 11612111467 & NaNaFeNaCaNaNaNaAlFeSn \\
\hline 6597114103 & FeZnHSnNaNaAlNaKMg \\
\hline 9711511299 & HSnNaNaZnNaNaCaHH \\
\hline 85105108101 & PbZnNaKZnNaKPbNaKNa \\
\hline 11210410165 & NaNaCaNaKAlNaKNaFeZn \\
\hline 65108121115 & FeZnNaKPbNaCaNaNaNaZn \\
\hline 10310811065 & NaKMgNaKPbNaNaKFeZn \\
\hline 71108121115 & SnNaNaKPbNaCaNaNaNaZn \\
\hline 9711410365 & HSnNaNaAlNaKMgFeZn \\
\hline
\end{tabular}

Hence, the cipher would be -

NaNaFeNaCaNaNaNaAlFeSnFeZnHSnNaNaAlNaKMgHSnNaNaZnNaNaCaHHPbZnNaKZnNaKPbNaKNaNaNa CaNaKAlNaKNaFeZnFeZnNaKPbNaCaNaNaNaZnNaKMgNaKPbNaNaKFeZnSnNaNaKPbNaCaNaNaNaZnHSn NaNaAlNaKMgFeZn

As the length of the cipher is too long, the repeated elements are added, as stated below:

2NaFeCa3NaAlFeSnFeZnHSn2NaAlNaKMgHSn2NaZn2NaCa2HPbZnNaKZnNaKPbNaK3NaCaNaKAlNaKNaFe ZnFeNaKPbNaCa3NaZnNaKMgNaKPb2NaKFeZnSn2NaKPbNaCa3NaZnHSn2NaAlNaKMgFeZn

\section{B. Decryption -}

For decryption purpose, the reverse order operations of the proposed algorithm is ensured and operated to fetch the plain text from the cipher text.

\section{CONCLUSION}

Whenever the term safety comes in intellect and initiative, security is synonymous, but from time to time implementing security mechanism(s) like cryptographic techniques, biometric methods, genetic algorithm, quick response code mechanisms, etc. has not only been sturdy but cost constrained as well. The design, implementation and incorporation of the cryptographic algorithm are the core of the conferred security amid predicament at bay like malicious intrusions. The proposed algorithm ensures the secured transmission modus operandi and thereby diminution in access time. The formulation of the paper ensures deliberations as well as elucidation on how the security methodology could be implemented and incorporated, employing an innovative cryptographic modus operandi.

\section{REFERENCES}

[1] Chowdhury, R., Datta, S., Dasgupta, S., De, M., “Implementation of Central Dogma Based Cryptographic Algorithm in Data Warehouse for Performance Enhancement”, International Journal of Advanced Computer Science and Applications, 6 (11), November, 2015, ISSN (Online)-2156 5570, ISSN (Print)-2158 107X, pp. 29-34

[2] Chowdhury, R., Dey, K., S., Datta, S., Shaw, S., "Design and Implementation of Proposed Drawer Model Based Data Warehouse Architecture Incorporating DNA Translation Cryptographic Algorithm for Security Enhancement”, Proceedings of International Conference on Contemporary Computing and Informatics, IC3I 2014, Organized by Sri Jayachamarajendra College of Engineering, Mysore, 
Proceedings in USB: CFP14AWQ-USB, ISBN-978-1-4799-6628-8, INSPEC Accession Number-14881472, Published and Archived in IEEE Digital Xplore, ISBN-978-1-4799-6629-5, pp. 55-60

[3] Chowdhury, R., Bose, R., Sengupta, N., De, M., "Logarithmic Formula Generated Seed Based Cryptographic Technique Using Proposed Alphanumeric Number System and Rubik Rotation Algorithm”, Proceedings of IEEE 2012 International Conference on Communications, Devices and Intelligent Systems, CODIS 2012, Organized by Jadavpur University, Kolkata, Proceedings in CD: IEEE Catalog NumberCFP1207U-CDR, ISBN-978-1-4673-4698-6, Proceedings in Print: IEEE Catalog Number-CFP1207U-PRT, ISBN-978-1-4673-4697-9, INSPEC Accession Number-13285714, Published and Archived in IEEE Digital Xplore, ISBN-978-1-4673-4700-6, pp. 564-567

[4] Chowdhury, R., Ghosh, S., De, M., "String Graphixification Based Asymmetric Key Cryptographic Algorithm Using Proposed Concepts of GDC and S-Loop Matrix”, Proceedings of IEEE/OSA/IAPR International Conference on Informatics, Electronics \& Vision 2012, ICIEV 2012, Organized by University of Dhaka, Dhaka, Bangladesh, Proceedings in CD: IEEE Catalog Number-CFP1244S-CDR, ISBN978-1-4673-1152-6, Proceedings in Print: IEEE Catalog Number-CFP1244S-PRT, ISBN-978-1-4673-1151-9, Conference Proceedings: ISSN-2226 2105, INSPEC Accession Number-13058551, Published and Archived in IEEE Digital Xplore, ISBN-978-14673-1153-3, pp. 1152-1157

[5] Chowdhury, R., Gupta, S., S., Saha, A., "Stochastic Seed Based Cryptographic Technique [SSCT] Using Dual Formula Key [DFK]", Proceedings of International Conference on Communication and Industrial Applications, ICCIA 2011, Science City, Kolkata, Proceedings in CD: IEEE Catalog Number-CFP1135R-CDR, ISBN-978-1-4577-1916-5, Proceedings in Print: IEEE Catalog Number-CFP1135R-PRT, ISBN-978-1-4577-1915-8, Published and Archived in IEEE Digital Xplore, ISBN-978-1-4577-1915-8, pp. 1-5

[6] Chowdhury, R., De, N., Ghosh, S., "Design and Implementation of RNS Model Based Steganographic Technique for Secured Transmission”, International Journal of Advanced Research in Computer Science and Software Engineering, 2012, 2 (3), ISSN22776451(P), ISSN-2277128X (O), pp. 132-136

[7] Chowdhury, R., Saha, A., Dutta, A., "Logarithmic Function Based Cryptosystem [LFC]", International Journal of Computer Information Systems, 2011, 2 (4), ISSN-22295208, pp. 70-76

[8] Chowdhury, R., Saha, A., Biswas, P., K., Dutta, A., "Matrix and Mutation Based Cryptosystem [MMC]”, International Journal of Computer Science and Network Security, 2011, 11 (3), ISSN-17387906, pp. 7-14

[9] Kahate, A., "Cryptography and Network Security”, Tata McGraw-Hill Education Pvt. Ltd., 2007, ISBN (10)-0-0706-4823-9, ISBN (13)-978-0-0706-4823-4

[10] Stallings, W., "Cryptography and Network Security Principles and Practices”, Prentice Hall, 2005, (13)-978-0-1318-7316-2

ISBN (10)-0-1318-7316-4, ISBN 